LA-UR-01-4597

Approved for public release; distribution is unlimited.

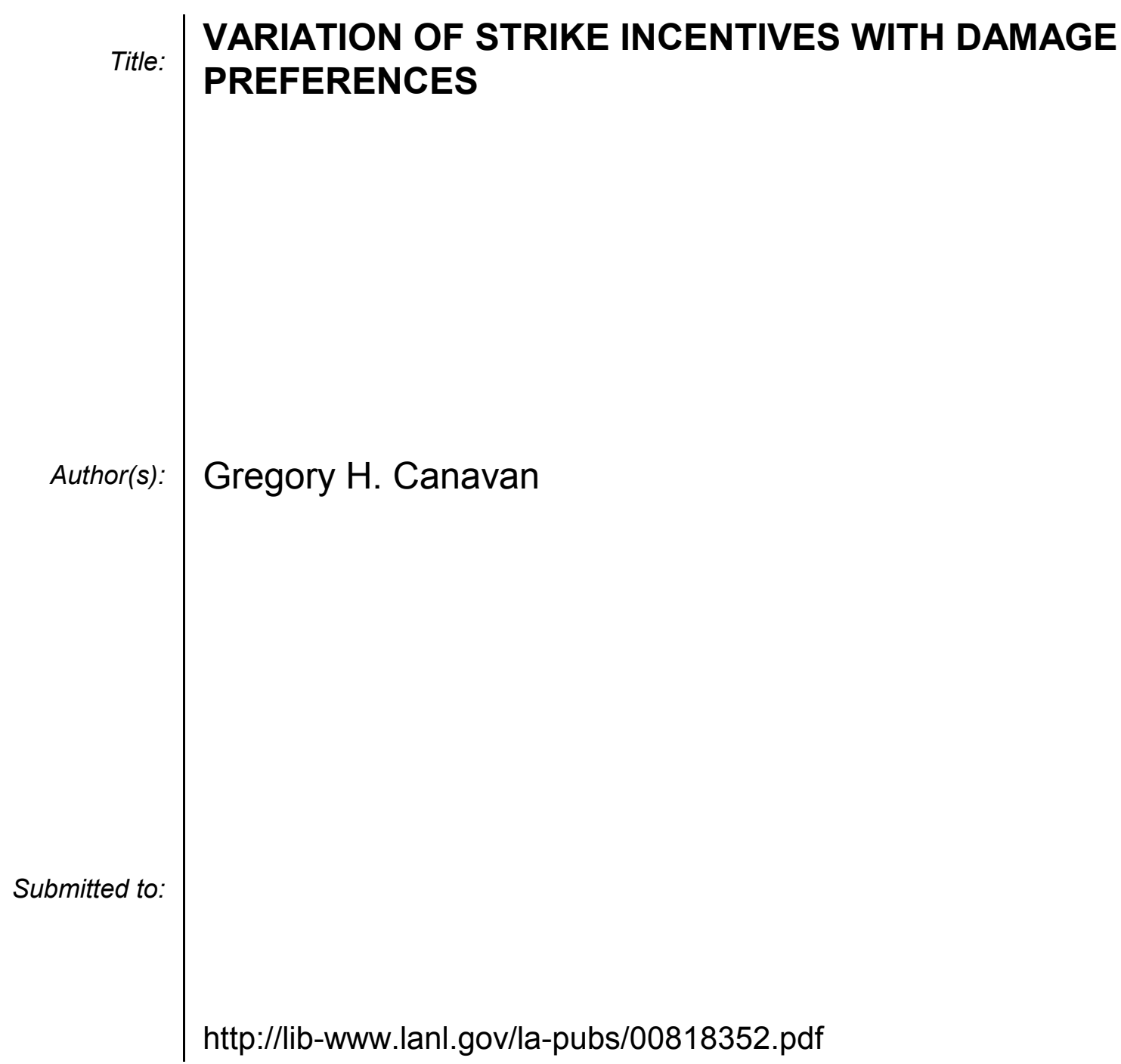

Los Alamos National Laboratory, an affirmative action/equal opportunity employer, is operated by the University of California for the U.S. Department of Energy under contract W-7405-ENG-36. By acceptance of this article, the publisher recognizes that the U.S. Government retains a nonexclusive, royaltyfree license to publish or reproduce the published form of this contribution, or to allow others to do so, for U.S. Government purposes. Los Alamos National Laboratory requests that the publisher identify this article as work performed under the auspices of the U.S. Department of Energy. Los Alamos National Laboratory strongly supports academic freedom and a researcher's right to publish; as an institution, however, the Laboratory does not endorse the viewpoint of a publication or guarantee its technical correctness. 


\title{
VARIATION OF STRIKE INCENTIVES WITH DAMAGE PREFERENCES
}

\author{
Gregory H. Canavan
}

If both sides prefer survival of high value over military targets and destruction, they do not see a net incentive to strike, because strike allocations vary little with damage preference while first strike costs vary directly with them.

This note studies the sensitivity of strike incentives to damage preferences, using the exchange and cost models derived and discussed in a companion report ${ }^{1}$ and force levels discussed in earlier papers. ${ }^{2}$ For moderate forces, strike allocations and magnitudes vary little with damage preference L, while first strike costs vary linearly with L. For nominal values reflecting a preference for the survival of high value targets over their destruction for high value over military targets, the costs of action are far greater than those of inaction over a wide range of values of military damage preferences. Thus, if both sides prefer survival of high value over military targets and destruction, they do not see a net incentive to strike, and crises are terminated by inaction.

Parameters. The principal inputs to the calculations are forces and preferences. It is assumed that $\mathrm{U}$ and $\mathrm{P}$ both have START III-like forces of 2,000 weapons, of which half are survivable. Each sides' objectives are represented by three preferences (L, K, V), where $\mathrm{L}$ is U's usual preference for damage to P's military relative to preventing damage to its own military targets, $\mathrm{K}$ is U's preference for survival of its own high value targets relative to survival of its own military targets, and V is U's preference for damaging P's high value targets relative to the survival of its own military targets, so that $\mathrm{V} / \mathrm{L}$ is $\mathrm{U}$ 's relative preference for damaging high value versus military targets.

None of these parameters are known with precision. It is generally assumed that a non-aggressive strong side could be characterized by $\mathrm{L} \approx 0.1$ to 0.25 and that more aggressive sides would be represented by larger $\mathrm{L}$. If $\mathrm{U}$ places high value on the survival of its high value or urban targets, it should have $\mathrm{K}>1$. If $\mathrm{U}$ is not interested in damaging the other's high value, it should have $\mathrm{V} \approx 0$. For nominal conditions, it is assumed below that both sides have preferences (L, 2, 1), where $\mathrm{L}$ is varied from 0.1 to 0.9 . Sensitivity to other values of $\mathrm{K}$ and $\mathrm{L}$ is discussed below. 
Through exchanges and the minimization of first strike costs, these parameters determine the two sides' allocations of first and second strikes to missiles, military, and high value targets. The process and generic results are discussed in the earlier report. Here, it suffices to recall that $(f, g, h)=$ fraction of $U$ (1st strike on missiles, second strike on military, 1st strike on military targets).

Results. Figure 1 shows the variation of allocations with L: $g \approx 2 / 3$ throughout; $h$ increases from 0.5 to 0.7 as $\mathrm{L}$ increases from 0.1 to 0.4 and then grows more slowly; $\mathrm{f}$ decreases from 0.5 to 0.25 in $0.3<\mathrm{L}<0.4$ as more aggressive opponents shift weapons from missiles to value to increase damage done.

Figure 2 shows the resulting first and second strikes, which reflect the above changes in allocations. At large L the top curve is the total first strike, F, which increases about $50 \%$ between $\mathrm{L}=0.3$ and 0.4 , reflecting the fall in $\mathrm{f}$ in Fig. 1. Most of that increase is in $F_{m}$, the strike on military targets. The first strike on value targets, $F_{v}$, falls slightly in accord with the increase in h shown in Fig. 1, which reflects the increased allocation of first strike to military targets at the expense of high value. Second strikes on value are essentially constant. Second strikes on military value increase, which produces an increase from 1,000 to 1,200 in total second strikes.

Figure 3 shows the components of first strike costs. Most vary little, but the cost of incomplete damage to the other's military, $\mathrm{C}_{1 \mathrm{mo}}$, and damage one's own value, $\mathrm{C}_{1 \mathrm{~ms}}$, have sequential increases, so their sum, the total damage on military value, $\mathrm{C}_{1 \mathrm{~m}}$, increases throughout, which causes the total cost, $\mathrm{C}_{1}$, to increase from 2.5 to 2.8. Much of $\mathrm{C}_{1}$ is due to damage to one's own value, $\mathrm{C}_{1 \mathrm{vs}} \approx 2$, but it does not vary with $\mathrm{L}$. Figure 2 shows that both sides allocate their strikes such that about 400 second strike weapons fall on high value targets. For $\approx 1 / \mathrm{v} \approx 100$ high value targets, that strike saturates the exponential in the damage function, so $\mathrm{C}_{1 \mathrm{vs}} \approx \mathrm{K}=2$, as seen.

Figure 4 shows the costs to $\mathrm{U}$ and $\mathrm{P}$ for reaching nodes 1 and 2, where $\mathrm{P}$ decides whether to strike first on the branch where $U$ has the option to preempt. The bottom curve is the cost of inaction, $\mathrm{L}+\mathrm{V}=\mathrm{L}+1$, which increases from 1.1 to 1.9. The top two curves are those for a strike by $\mathrm{U}$ followed by a restrike by $\mathrm{P}$. They show an interesting reversal; for $\mathrm{L}<0.3 \mathrm{P}$ 's cost is reduced if it lets $\mathrm{U}$ strike. However, the two top strikes curves are far above that for inaction, so the preferred choice at node 5 is inaction. As the forces and 
preferences are the same for both sides, inaction is also the preferred choice at nodes 3 and 4,6 , and 7 , so this configuration is seen as quite stable by both. From the above demonstration that $\mathrm{C}_{1} \approx \mathrm{C}_{1 \mathrm{v}} \approx \mathrm{C}_{1 \mathrm{vs}} \approx \mathrm{K}$ and the weak dependence of allocations on $\mathrm{K}$, it follows that stability is robust against excursions in $\mathrm{K}$ down to values at least on the order of unity. Sensitivity studies show that it is also insensitive to excursions in V.

Summary and conclusions. For START III level forces, strike allocations and magnitudes vary little with L, but first strike costs vary directly with L, which means that for $\mathrm{K}$ reflecting a preference for the survival of high value targets over their destruction and a preference for high value over military targets, the costs of action are far greater than those of inaction for a wide range of values of damage preference L. Thus, if both sides have much greater preferences for the survival of their high value targets than for military targets or destruction, they do not see a net incentive to strike, and crises are terminated by inaction. Recent decades suggest strong preferences for the survival of high value and that this has contributed to the lack of direct conflict during that period.

\section{References}

\footnotetext{
${ }^{1}$ G. Canavan, "Cost of Addressing Targets of Unequal Value," Los Alamos National Laboratory Report LA-UR-01-draft, June 2001.

${ }^{2}$ G. Canavan, "Analysis of Decisions in Bi- and Tri-Lateral Engagements," U.S. State Department Stability Workshop (Institute for Defense Analysis, November 2000); Los Alamos National Laboratory Report LA-UR-00-5737, November 2000.
} 
Fig. 1. Allocations of first and second strikes to missiles, military, and high value targets.

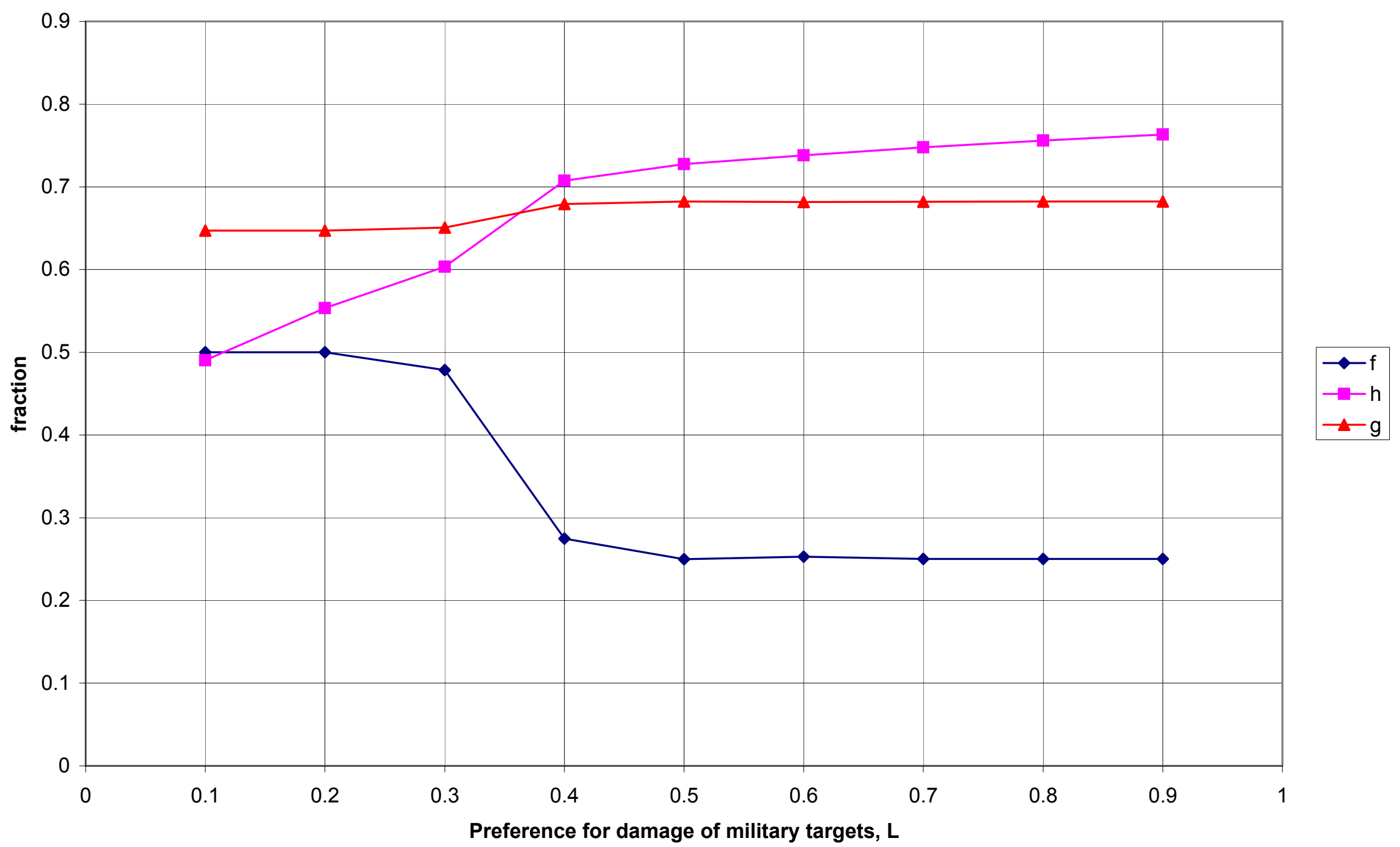


Fig. 2. First and second strikes

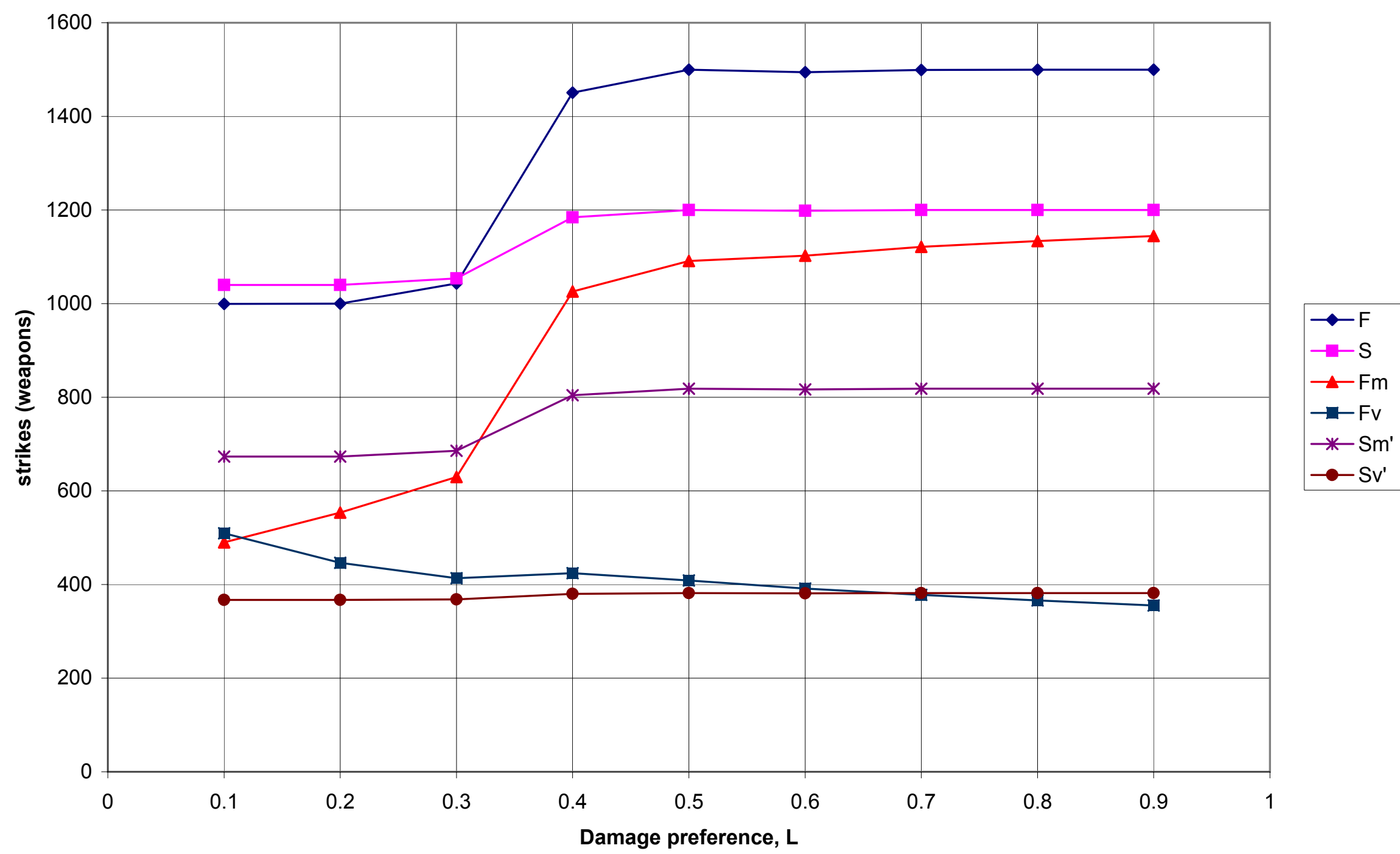


Fig. 3. First strike costs

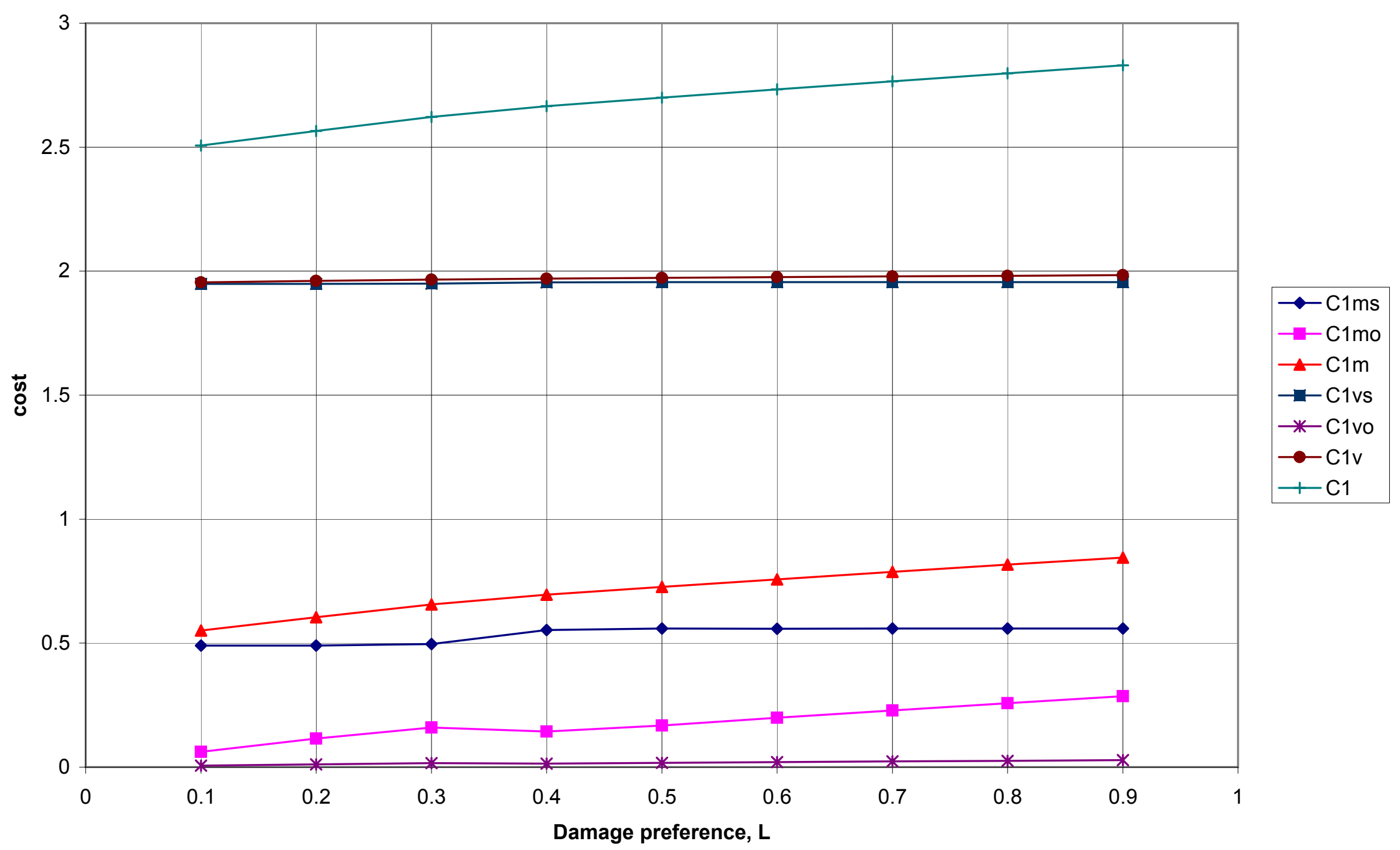


Fig. 4. Costs of nodes $1 \& 2$ as functions of preference for damage to military targets $\left(\mathrm{K}=\mathrm{K}^{\prime}=2 ; \mathrm{V}=\mathrm{V}^{\prime}=1\right)$

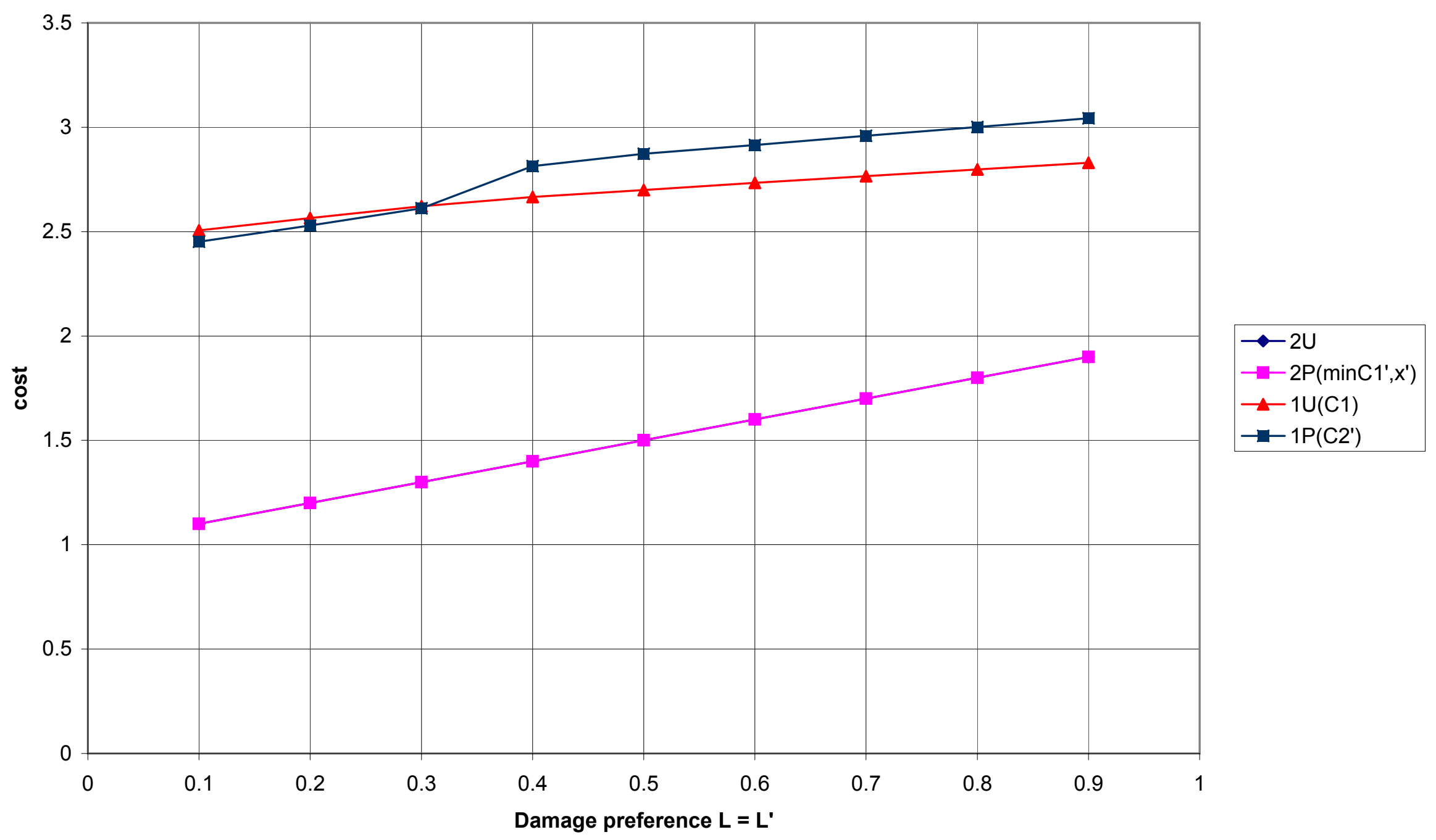


node $5 f$

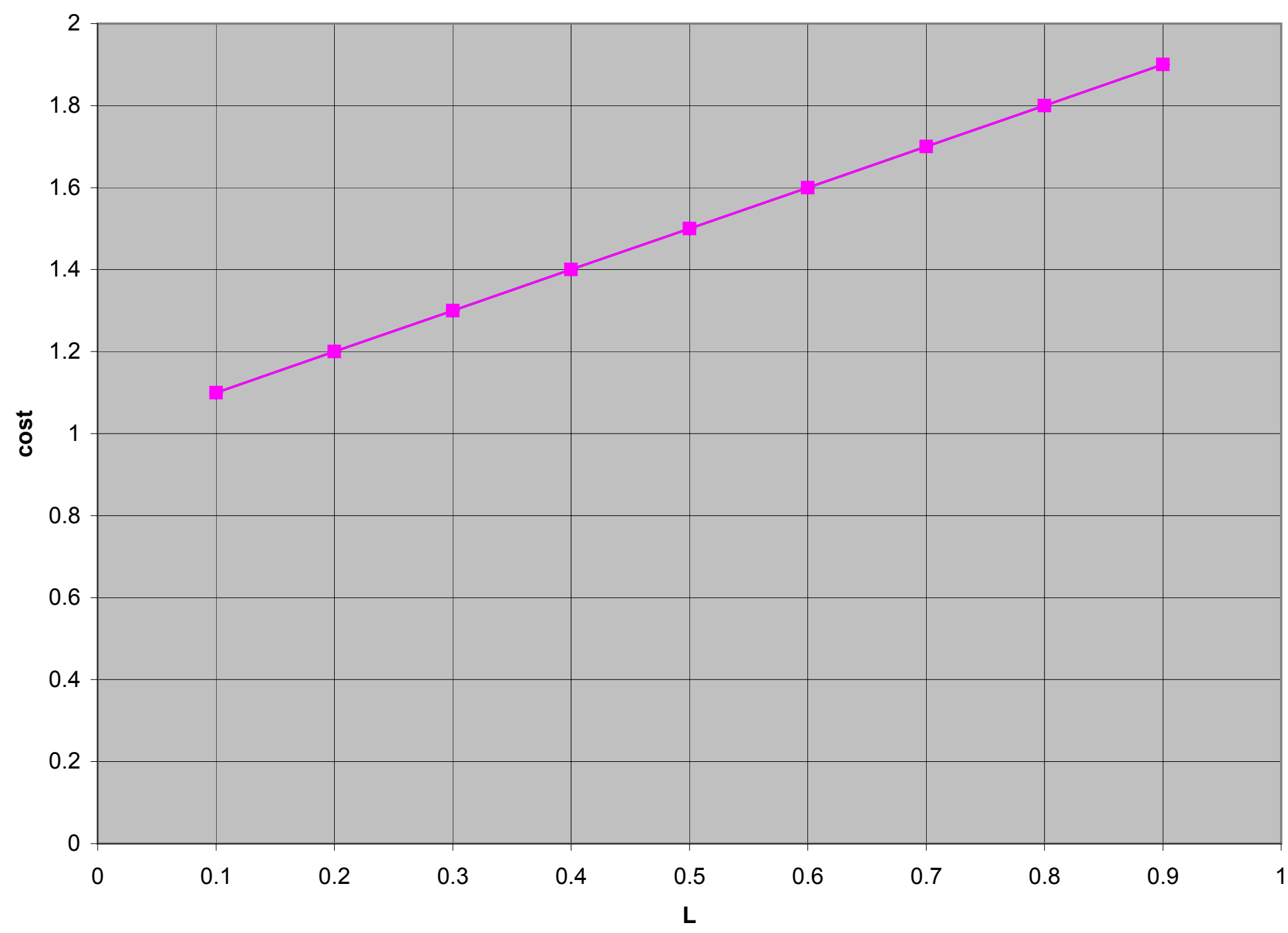

$\multimap-5 \mathrm{U}(\min C 1,2 \mathrm{U})$

$-5 \mathrm{P}$ 
node 7

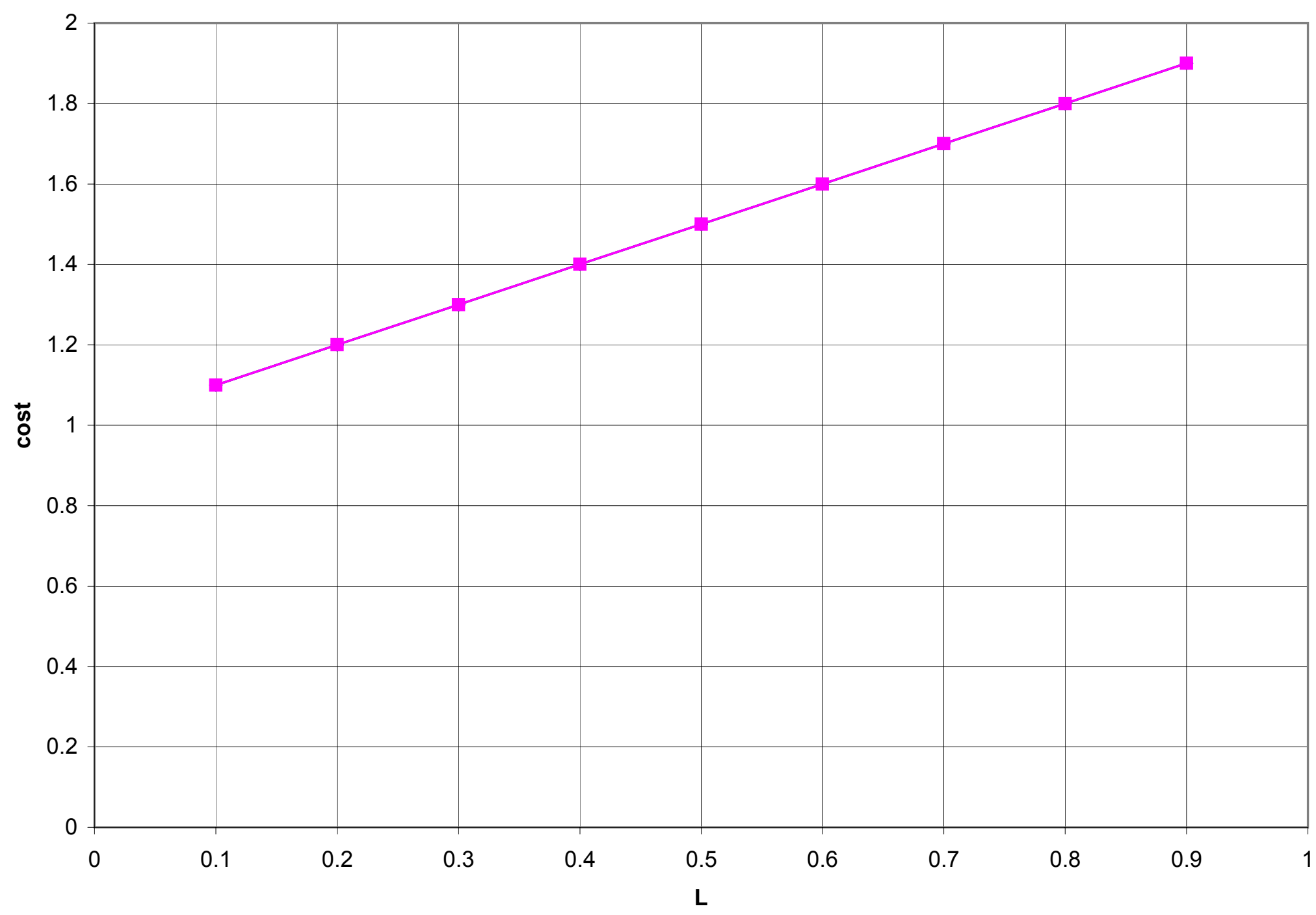

$\bullet 7 \mathrm{U} u=1 / 2$ 


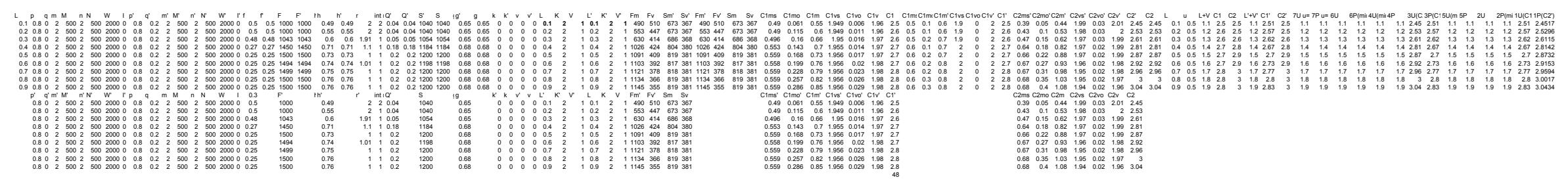

